\title{
Correcting the side effects of ADC filtering in MR image reconstruction
}

\author{
Carole Lazarus, Maximilian März \& Pierre Weiss *
}

May 14, 2020

\begin{abstract}
This work investigates the role of the filters implemented on Analog-to-Digital Converters for the reconstruction of magnetic resonance images. We analyze the effects of these filters both from a theoretical and an experimental point of view and demonstrate how it may lead to severe degradation of the reconstructed images when the distance between consecutive samples is larger than Shannon's limit. Based on these findings, we propose a mathematical model and a numerical algorithm that allow to mitigate such filtering effects both for linear and nonlinear reconstructions. Experiments on simulated and real data on a 7 Tesla scanner show that the proposed ideas allow to significantly improve the overall image quality. These findings are particularly relevant for high resolution imaging and for recent sampling schemes saturating the maximum gradient amplitude. They also open new challenges in sampling theory.
\end{abstract}

\section{Introduction}

The standard acquisition model in magnetic resonance imaging (MRI) states that the Fourier transform of an image $u: \Omega \rightarrow \mathbb{C}$ is sampled on a set of discrete points, in what is commonly called the $k$-space. The set $\Omega \subset \mathbb{R}^{d}$ is the field of view, with $d=2$ or $d=3$ denoting the space dimension. A particularity of MRI is that these samples are measured along several parametrized curves $\lambda:[0, T] \mapsto \mathbb{R}^{d}$, called $k$-space trajectories or shots. Given a sampling period $\Delta t$ and letting $\hat{u}$ denote the Fourier transform of the image $u$, the measured samples are therefore modeled as

$$
y_{j}=\hat{u}(\lambda(j \Delta t)),
$$

where $j \in \mathbb{N}$ denotes the index of the measurement [17]. Most commonly, these points lie on a grid, which is filled by parallel Cartesian lines [24], but non-Cartesian sampling such as spiral trajectories [35] may also be used for their greater sampling efficiency and are becoming increasingly popular with the advent of compressed sensing [31].

The model expressed in equation (1) does not account for the analog-to-digital converter (ADC), which converts the continuous signal $f(t)=\hat{u}(\lambda(t))$ into discrete values. The model that describes this process is a linear time invariant filter of the type:

$$
y_{j}=(h \star f)(j \Delta t),
$$

where $\star$ denotes a convolution operator and $h$ is a filter that depends on the ADC technology. The main contribution of this article is twofold: First, we demonstrate that neglecting the filtering effect in model (2) can have a dramatic impact on the reconstruction quality for large sampling periods $\Delta t$. Secondly, numerical algorithms are proposed that allow to handle the unwanted side-effects caused by the filtering.

We give particular attention to two typical situations: a simple integrator ADC and more advanced bandlimiting filters which are used in modern MRI scanners.

- Integration effect. The earliest ADCs were integrating ADCs, meaning that the ADC simply integrates the Fourier transform $\hat{u}$ along the $k$-space path $\lambda$. This model reads as $y_{j}=\int_{(j-1) \Delta t}^{j \Delta t} \hat{u}(\lambda(t)) d t$ and can be cast into equation (2) by choosing $h=\mathbb{1}_{[0, \Delta t]}$. The interest of choosing a large sampling period $\Delta t$ is to increase the

*P. Weiss is a CNRS researcher both at ITAV and IMT, Université de Toulouse and CNRS, email: pierre.armand.weiss @ gmail.com. 
signal-to-noise ratio (SNR) since more signal is averaged over time [20], which is a typical reasoning in MRI physics. We will see later that this benefit might not compensate the downsides that come with it.

- Bandlimiting filtering. On most MR scanners, the ADC bandwidth reaches several megahertz, while the receiver bandwidth usually ranges from about 5 to $500 \mathrm{kHz}[4,18]$. To produce the measurements at the desired sampling rate, the digitized data is filtered before decimation [21, 5, 40]. This bandlimiting step can be expressed as (2) and has different purposes [21, 22]: i) it discards the spurious noise and high frequencies from the free induction decay signal and ii) it allows to avoid aliasing of the temporal signal $f$. In particular, when using Cartesian trajectories, wraparound effects are prevented by cutting of the signal which is outside of the field of view in the readout direction $[34,30]$, as will be seen in the next section. A perfect bandlimiting filter takes the form $h(t)=\operatorname{sinc}(t / \Delta t)$.

To the best of our knowledge, the effects of ADC filtering on MR image reconstruction for non Cartesian trajectories have only been described scarcely in the literature. The manufacturers design these filters based on Cartesian sampling. This results in filters that may be unadapted to other sequences. In the mathematics community, we are not aware of a single paper in sampling theory which accounts for them. More generally, we are not aware of any reference that takes this effect into account in a reconstruction algorithm. We came across this phenomenon while trying to reconstruct real MR images and it took us a lot of time and experiments to disentangle the possible sources of reconstruction errors. The purpose of this paper is to illustrate the consequences of these filters, to provide criteria when they need to be accounted for, to show how they can be estimated in practice and how their effect can be mitigated using adapted reconstruction algorithms. The proposed algorithms are then tested against simulated and real data, showing significant improvement in the reconstruction quality, especially when using the novel trajectories designed in the framework of compressed sensing.

\section{Notation}

We assume that the image domain is $\Omega=[-1 / 2,1 / 2]^{2}$. We chose to work in $2 \mathrm{D}$ to simplify the exposition, but an extension to 3D is direct. The domain can be shifted and inflated to account for a different field of view with scaling arguments. Let $u: \Omega \rightarrow \mathbb{C}$ denote a magnetic resonance image in $L^{2}(\Omega)$. Its Fourier transform is defined for all $\xi \in \mathbb{R}^{2}$ by

$$
\hat{u}(\xi)=\int_{\Omega} \exp (-2 \iota \pi\langle x, \xi\rangle) u(x) d x .
$$

Notice that with the choice $\Omega=[-1 / 2,1 / 2]^{2}$, Shannon's sampling theorem [39] suggests to sample the Fourier transform on a Cartesian grid, with grid size of length less than 1 .

The notation $\delta$ stands for the Dirac delta function at 0 , while $\delta_{x}$ stands for the Dirac delta function at a position $x$. Given two functions $f$ and $g$, the tensor product of $f$ and $g$ is defined by $(f \otimes g)(x, y)=f(x) \cdot g(y)$ for all $x, y$. We recall that the Fourier transform preserves the tensor form, i.e. $\mathcal{F}(f \otimes g)=\mathcal{F}(f) \otimes \mathcal{F}(g)$.

\section{The deleterious consequences of filtering}

The aim of this section is to describe the effects of ADC filtering on image reconstructions and to ascertain when it is important to account for them.

\subsection{The case of Cartesian sampling}

The filtering effect in the case of Cartesian sampling with constant speed $s$ can be understood by an analytical argumentation. In that case, for each sampling point, the filtering is performed along the readout direction only. A convenient way to formalize this observation is to introduce the tensor product filter $\hat{\mu}=g \otimes \delta$, where $g(t)=h(t / s)$ and to observe that the acquisition model (2) can then be written as

$$
y_{i, j}=(\hat{u} \star \hat{\mu})\left(i \Delta \xi_{1}, j \Delta \xi_{2}\right)
$$


where $(i, j) \in\{-n / 2, n / 2-1\}^{2}$ describe the set of sample indices, $\Delta \xi_{1}, \Delta \xi_{2}$ denote the grid spacing in the Fourier domain, and $n \in 2 \mathbb{N}$ is the number of samples in each $k$-space direction.

Now, by not accounting for the filtering effect, the best we can hope for is to reconstruct an image $u^{*}$ of the form $u^{*}=\mathcal{F}^{-1}(\hat{u} \star \hat{\mu})=u \cdot \mu$, where $\mu$ is given by

$$
\mu=\mathcal{F}^{-1}(g \otimes \delta)=\mathcal{F}^{-1}(g) \otimes \mathcal{F}^{-1}(\delta)=\mathcal{F}^{-1}(g) \otimes \mathbb{1} .
$$

Depending on the filter $h$, different effects can be expected.

Integration. In the case of an integration filter, we have $h=\mathbb{1}_{[0, \Delta t]}$, where $\Delta t$ is the sampling period. Hence, we get

$$
\left|u^{*}\right|(x, y)=s \Delta t|u(x, y) \operatorname{sinc}(s \Delta t x)| .
$$

The filtering effect produces an image modulated by a sinc. Depending on the spacing between samples $s \Delta t$, this effect will either just lower the contrast at the image boundaries or create low frequency oscillations.

Sinc. By using a sampling period $\Delta t$, the standard Shannon-Nyquist sampling theorem states that it is impossible to reconstruct frequencies beyond the interval $I=\left[-\frac{1}{2 \Delta t}, \frac{1}{2 \Delta t}\right]$. The usual way to avoid aliasing effects, is to use a perfect bandlimiting filter of the type $\hat{h}=\mathbb{1}_{I}$. In that case, we would get $h(t)=\operatorname{sinc}\left(\frac{t}{\Delta t}\right)$ and

$$
\left|u^{*}\right|(x, y)=s \Delta t|u(x, y)| \mathbb{1}_{\left[-\frac{1}{2 s \Delta t}, \frac{1}{2 s \Delta t}\right]}(x) .
$$

The filtering effect in that case simply clips the image in one direction. This effect can be used to avoid aliasing of the out of field of view image contents [34, 30, 28].

We now propose to simulate the effect using 4 different Cartesian sequences with $\Delta \xi=s \Delta t \in\left\{\frac{1}{2}, 1,2,4\right\}$. The value $\Delta \xi=1 / 2$ corresponds to the most standard Cartesian trajectory in MRI (oversampling factor of 2 along the readout direction). The value $\Delta \xi=1$ corresponds exactly to the Shannon's limit sampling rate for an image supported on $[-1 / 2,1 / 2]^{2}$.

The measurements are simulated by incorporating the filtering effect as expressed in equation (3). The reconstruction algorithm is based on a standard inverse discrete Fourier transform (which does not account for any filtering effects). Fig. 1 and 2 show the results for the integration and the sinc filtering respectively as well with a cross section of the images. The modulation due to the integration filtering can be seen on the cross sections for all values of $\Delta \xi$. The effect of the sinc filtering is only observable for $\Delta \xi \in\{2,4\}$ : the disk is clipped with an irreversible loss of information. In MRI, this property is sometimes seen as beneficial since it avoids aliasing in the readout direction $[34,30,28]$. It can also be used to focus on smaller region of interest.

\subsection{The case of arbitrary trajectories}

For more general trajectories, it is difficult to derive an analytical description of the filtering effects. The proposed sampling model can be expressed as

$$
y_{i}=\int \hat{u} d \mu_{i}
$$

where $d \mu_{i}$ is a singular measure supported on a 1-dimensional curve. To the best of our knowledge, this problem has not been studied in the literature yet. A closely related issue was investigated by Aldroubi in [3]. Therein, he addresses the question of the existence of a stable linear reconstruction algorithm for bounded measures $\mu_{i}$ with compact support, two conditions that are not met in our setting. In what follows, we therefore restrict ourselves to provide experimental simulations based on spiral trajectories, which are one of the most widespread non-Cartesian trajectories.

Fig. 3 shows a sampling pattern made of 200 interleaved spirals, which were designed using the method proposed in [27]. Each spiral is depicted with a different color going continuously from blue to green. The number of samples is equal to 153600 , while the reference image contains $512 \times 512$ pixels: this corresponds to a subsampling factor of 1.7. Notice that while the distance between consecutive samples is about 1 pixel in the $k$-space center, it reaches about 5 pixels in the outer part, meaning that the filtering effect will have a higher impact on high frequencies. Images of a brain phantom [19] were reconstructed using a total variation based, nonlinear reconstruction algorithm described in 


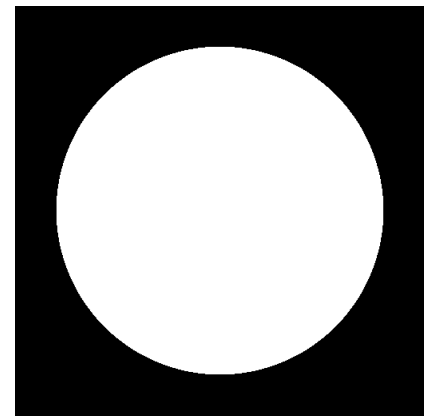

(a) Ground truth

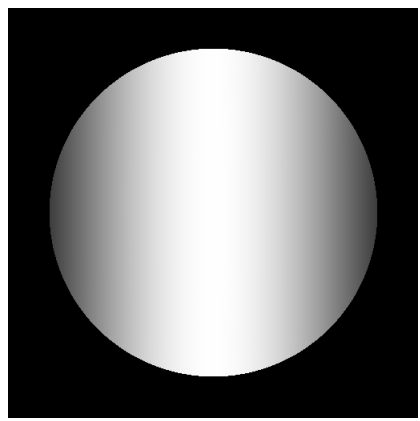

(d) $\Delta \xi=2$

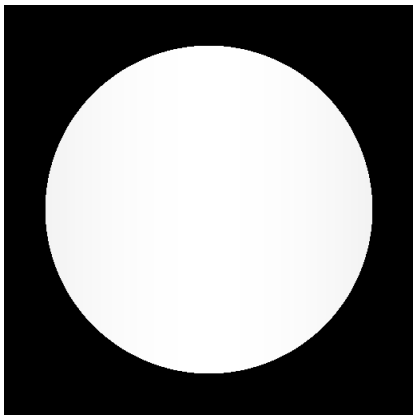

(b) $\Delta \xi=1 / 2$

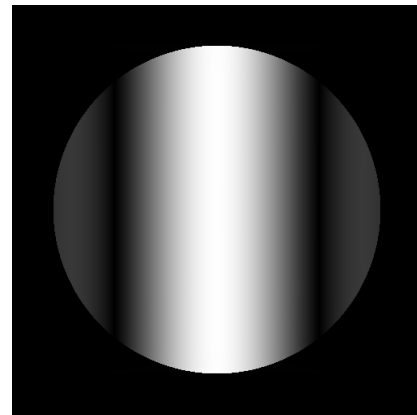

(e) $\Delta \xi=4$

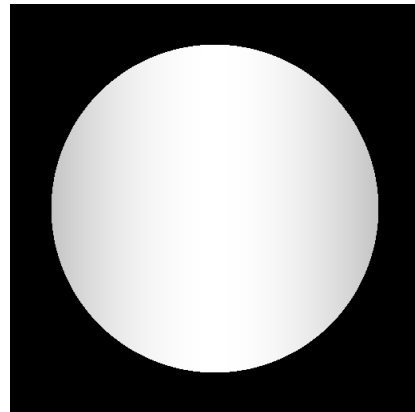

(c) $\Delta \xi=1$

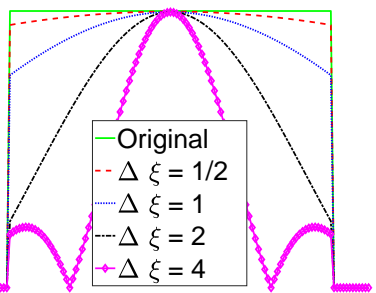

(f) Cross sections

Figure 1: The integration effect with a Cartesian sampling. Note: the images might be complex valued, and we only display their modulus. This explains why negative oscillations are seen as positive values.

Section 4.3.3. Fig. 4 shows the consequences of the integration and the sinc filtering on the images acquired with the spiral trajectory. In case of integration (Fig. 4e), the reconstructed image suffers from severe artifacts including a space varying blur, some contrast losses, a slight rotation (visible only when superimposing ground truth and reconstruction) and a modulation. In the case of bandlimiting filtering (Fig. 4f), the image quality degrades even more: a magnified region shows the loss of resolution as compared to the ground truth (Fig. 4d).

\subsection{Experimental validation of the filtering effects}

To verify the expected effects of the ADC, an ex vivo baboon brain was imaged using a gradient recalled echo (GRE) sequence to acquire Cartesian and spiral data.

\subsubsection{The trajectories and sampling steps}

In the case of Cartesian sampling, we set $\delta t=10 \mu \mathrm{s}$ with a field-of-view (FOV) of $20 \times 20 \mathrm{~cm}^{2}$ for a target resolution of $256 \times 256$. We then designed three trajectories composed of 256 lines with different sampling periods $\Delta t \in$ $\{\delta t, 2 \delta t, 8 \delta t\}$. When considering a normalized FOV, those downsampling factors correspond to a grid spacing in the $k$-space of $\Delta \xi=\frac{1}{2}, \Delta \xi=1$ and $\Delta \xi=4$ respectively. For the case $\Delta \xi=4$, we interleaved four sets of trajectories to fill the whole Cartesian grid, i.e. sampling the Fourier transform at Shannon's rate.

In the case of spiral trajectories, we set $\delta t=5 \mu \mathrm{s}$ with the same field-of-view (FOV) of $20 \times 20 \mathrm{~cm}^{2}$. Spirals were designed using the method proposed in [27] and were made of 20 shots for a target resolution of $512 \times 512$. In that experiment, we also studied the cases $\Delta t \in\{\delta t, 2 \delta t, 8 \delta t\}$. For the largest sampling period, we interleaved 4 spirals to measure as much information as for $\Delta t=2 \delta t$. 


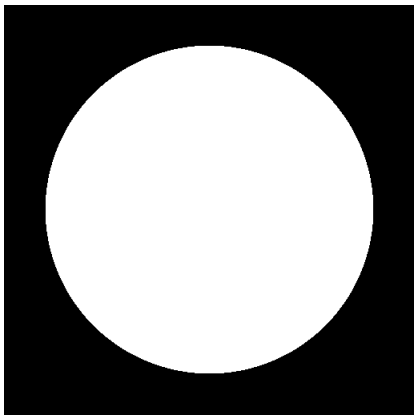

(a) Ground truth

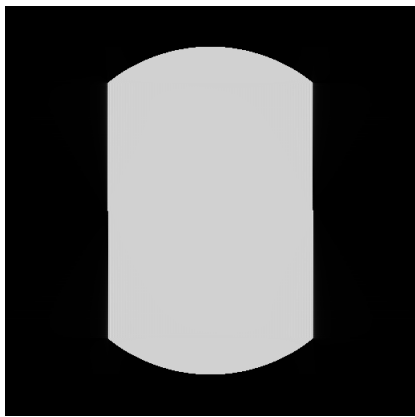

(d) $\Delta \xi=2$

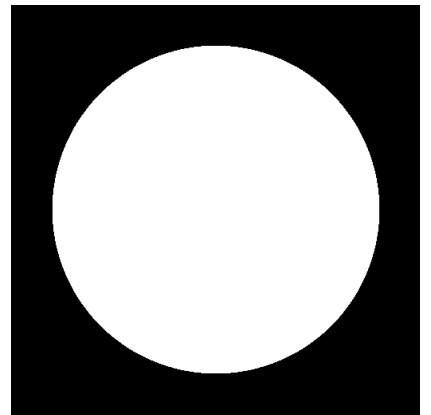

(b) $\Delta \xi=1 / 2$

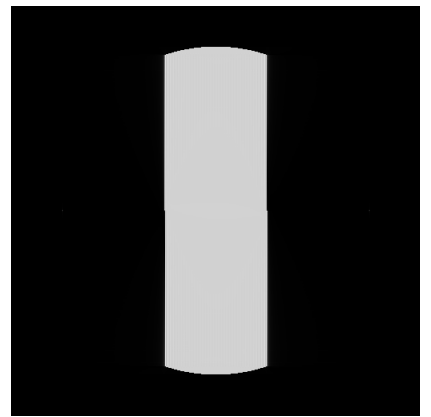

(e) $\Delta \xi=4$

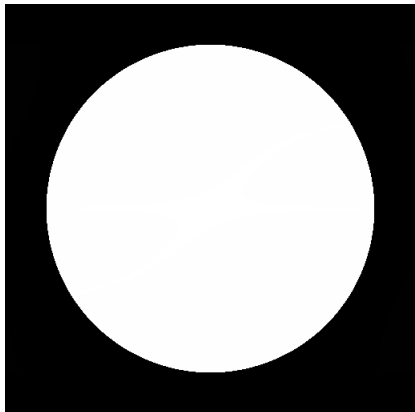

(c) $\Delta \xi=1$

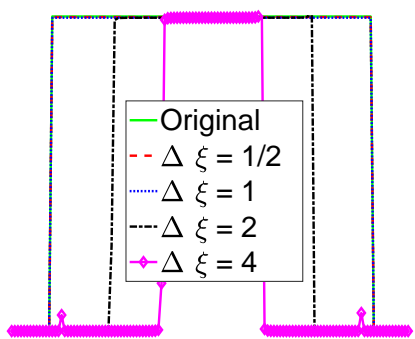

(f) Cross sections

Figure 2: The sinc filtering effect with Cartesian sampling. Note: in this experiment, some ringing appears on the boundaries, explaing why the clipped images are darker.

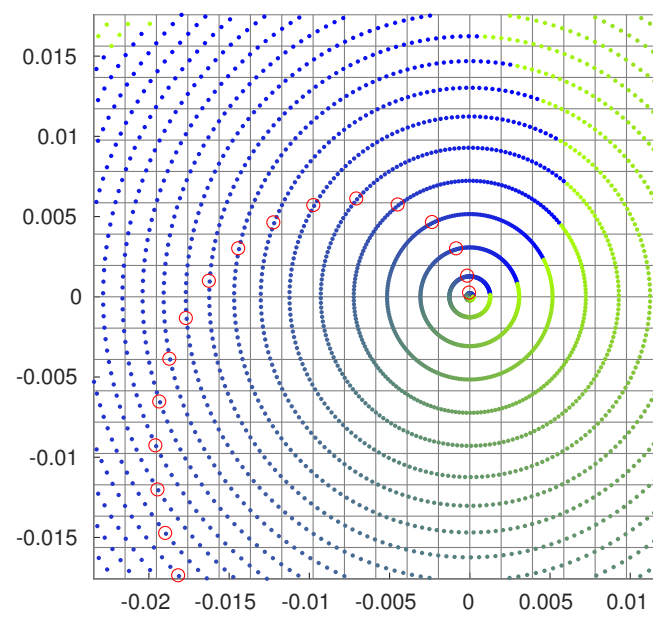

(a) Center

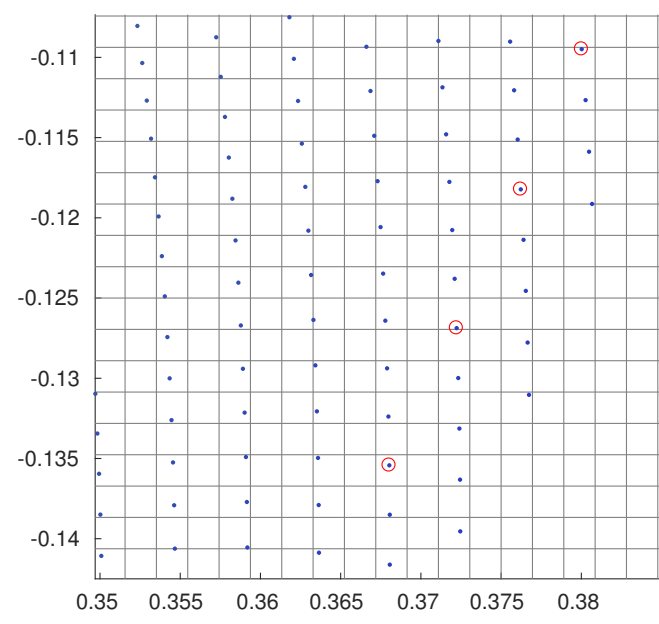

(b) Boundary

Figure 3: Two zooms on a sampling pattern made of 200 interleaved spirals. Each spiral has a different color.

\subsubsection{Filtering effects on experimental data}

The reconstruction results using a standard conjugate gradient algorithm are displayed in Fig. 5. 


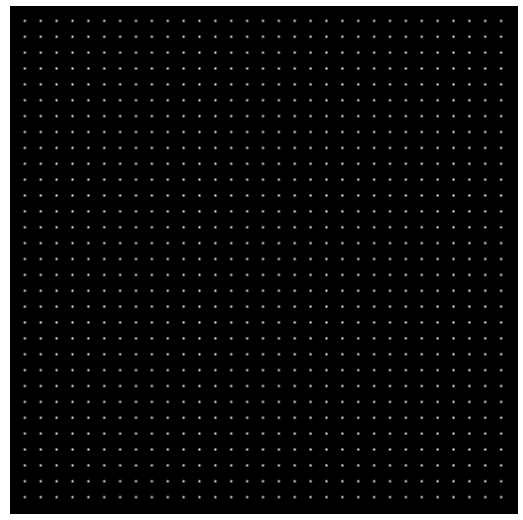

(a) Ground truth

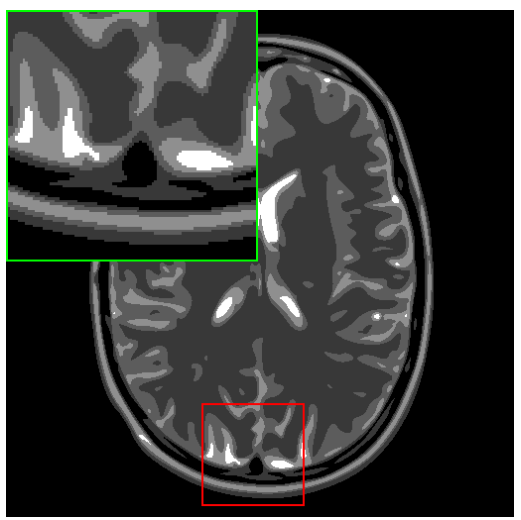

(d) Ground truth

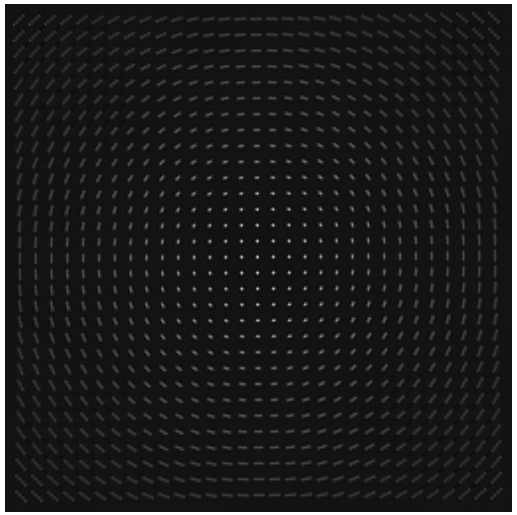

(b) Recon (int)

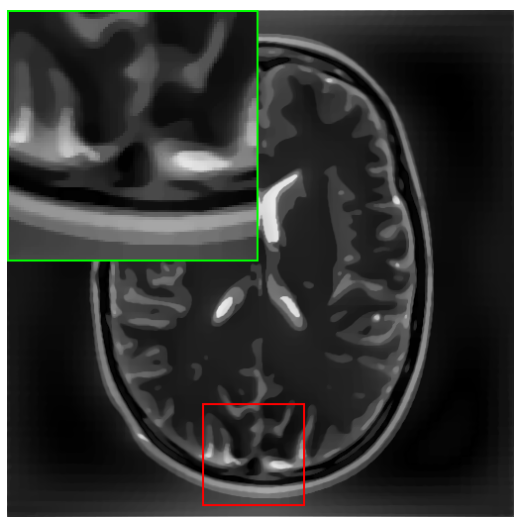

(e) Recon (int)

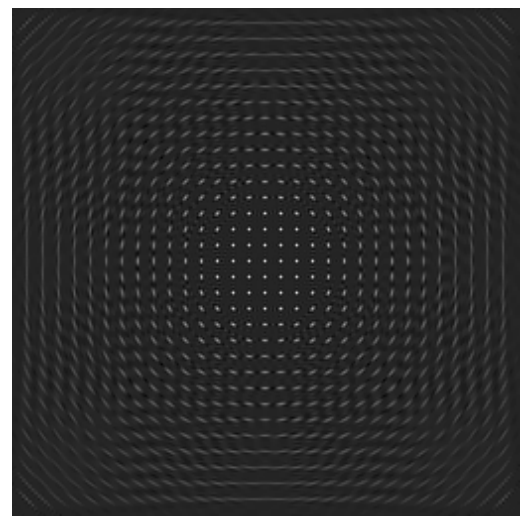

(c) Recon (sinc)

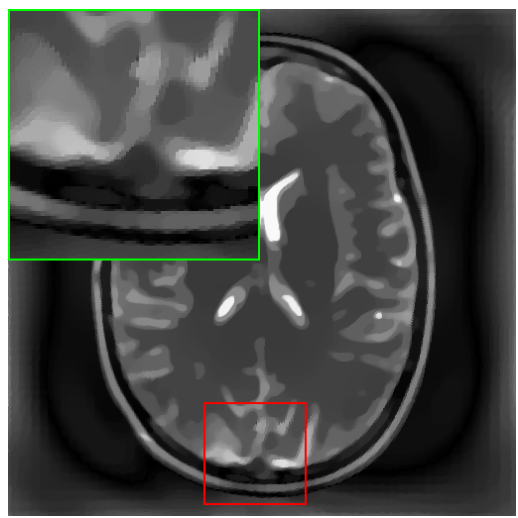

(f) Recon (sinc)

Figure 4: Example for reconstructions of an image sampled along the spirals shown in Fig. 3. The total variation based reconstruction algorithm does not account for the filtering effect. Top: the reconstruction of a Dirac comb illustrates the impulse response of the reconstruction algorithm. As can be seen, both the integrating filter (int) and the perfect bandlimiting filter (sinc) smooth out the image by integrating it along pieces of arcs with a width that increases radially. Bottom: smoothing effects of both filters can be observed on a phantom image.

In the case of Cartesian trajectories, no filtering effects are observed for $\Delta \xi=\frac{1}{2}$ (Fig. 5a). For $\Delta \xi=1$, corresponding to a sampling pattern at Shannon's rate, two black bands appear at the top and bottom of the image (Fig. 5b). For the image on Fig. 5c corresponding to $\Delta \xi=4$, four horizontal black bands can be observed and the object is cropped along the readout direction. This perfectly matches the effects described in Section 2.1 (Fig. 1 and 2), confirming that the MR scanner applies a bandlimiting filter on the temporal signal.

In the case of spiral sampling, the image corresponding to $\Delta t=5 \mu \mathrm{s}$ on Fig. $5 \mathrm{~d}$ is near perfectly resolved. For $\Delta t=10 \mu \mathrm{s}$, the image gets slightly more blurry and the contrast is slightly deteriorated. For $\Delta t=40 \mu \mathrm{s}$, the effect gets disastrous, with some parts of the image disappearing and strong rotational blurs.

The experiments provided in this section reveal that the usual ADC filters produce effects that can be neglected from a perceptual viewpoint when the maximal distance between consecutive samples is below half the Nyquist limit. The effect becomes visible starting from the Nyquist rate and becomes dramatic at twice this limit. 


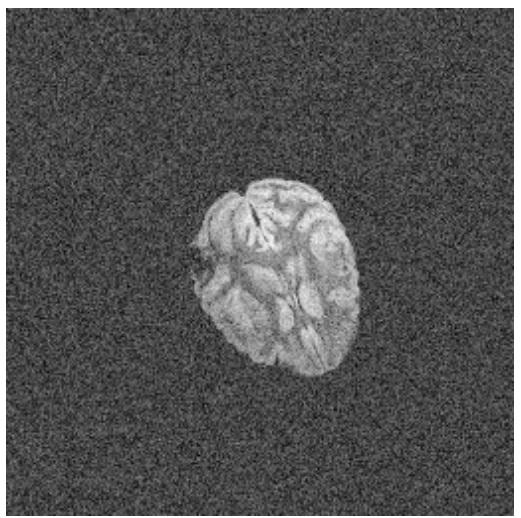

(a) $\Delta \xi=1 / 2$

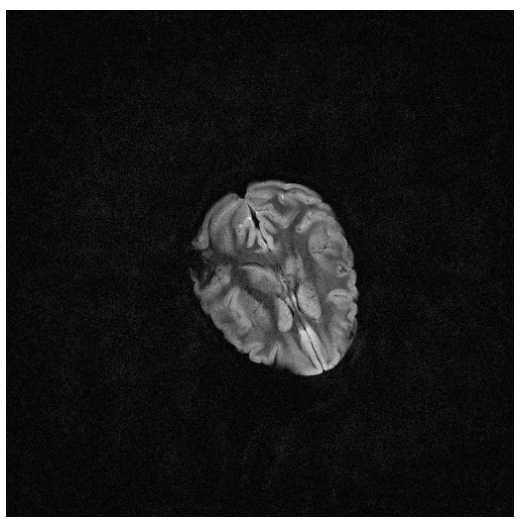

(d) $\Delta \xi=1 / 2$

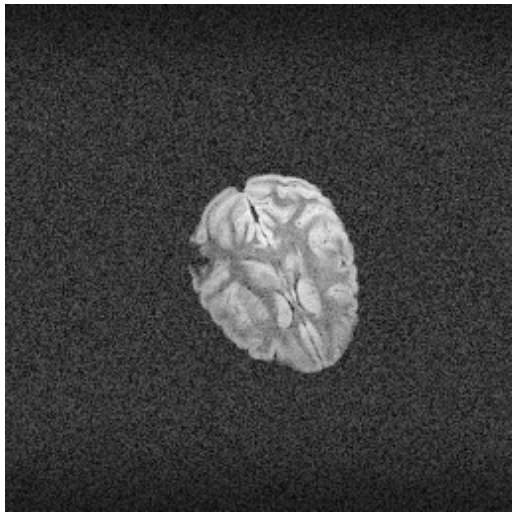

(b) $\Delta \xi=1$

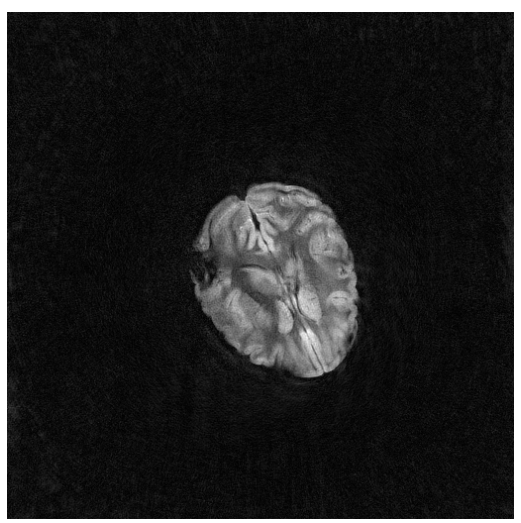

(e) $\Delta \xi=1$

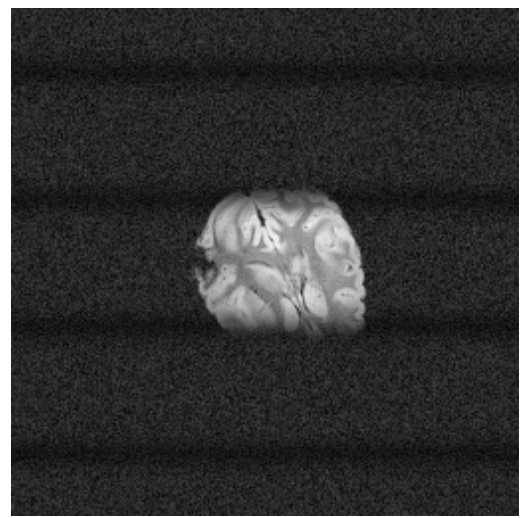

(c) $\Delta \xi=4$

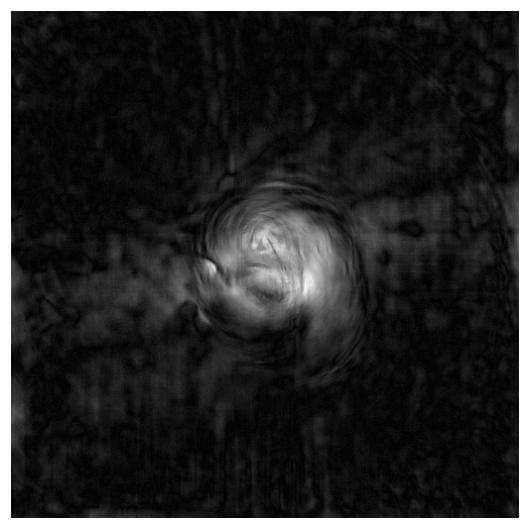

(f) $\Delta \xi=4$

Figure 5: Direct reconstruction results of experimental data for the Cartesian trajectories with a linear reconstruction algorithm (top) and spiral trajectories with a total variation based reconstruction (bottom). For each trajectory, we used different sampling periods. Note that we adapted the contrast to better highlight the effect.

\section{Estimating the filters}

The way the signal is digitized and processed depends on the MR receiver hardware, with specifications that are usually not transparent to the user. In the following part, we therefore design a reverse engineering technique to estimate the filter $h$.

\subsection{A filter estimation procedure}

The principle reads as follows:

- Set a sampling period $\delta t$, a downsampling factor $p \in \mathbb{N}$, a number of measurements $m \in p \mathbb{N}$ and a trajectory $\lambda:[0, m \delta t] \rightarrow \mathbb{R}^{2}$.

- Generate a first set of measurements $\mathbf{y}_{0} \in \mathbb{C}^{m}$ by sampling $f=\hat{u} \circ \lambda$ with the rate $\Delta t=\delta t$.

- Generate a second set of measurements $\mathbf{y}_{1} \in \mathbb{C}^{m / p}$ by sampling $f$ with the rate $\Delta t=p \delta t$.

The proposed filtering model (2) can be conveniently expressed by setting

$$
\mathbf{y}_{1}=\left(\mathbf{h} \star \mathbf{y}_{0}\right) \downarrow p,
$$


where $\star$ denotes the convolution product and $\downarrow p$ is the downsampling by a factor $p$.

Recovering $\mathbf{h}$ knowing $p, \mathbf{y}_{0}$ and $\mathbf{y}_{1}$ is a difficult calibration problem. It amounts to finding a linear time invariant operator that matches the two sets of measurements $\mathbf{y}_{0}$ and $\mathbf{y}_{1}$. Without the decimation operator $\downarrow p$, it would boil down to a deconvolution problem, which is usually ill-posed $[41,36]$. The decimation makes the problem even harder. This being said, a few elements simplify the problem. First, the calibration sequence is computed off-line once for all. Hence, the same trajectory can be played multiple times in order to improve the signal-to-noise-ratio. Moreover, the trajectory played to calibrate the system can be chosen so as to improve the identifiability of the filter $\mathbf{h}$. Ideally, it should stay close to the $k$-space center, where a lot of signal is available. In addition, it should sweep a large range of frequencies to help evaluating the action of the filter at every frequency. A simple and popular trajectory complying with these criteria, is a linear chirp, i.e. a function of the form $\lambda(t)=\left(k_{\max } \sin \left(\omega t^{2}\right), 0\right)$ for some frequency $\omega$ and small amplitude $k_{\max }$ [1]. This trajectory might not be feasible for a large range $k_{\max }$ or frequency $\omega$. It is possible to project it onto the set of admissible trajectories using the algorithm proposed in [13], see Fig. 6a. The same trajectory can then be rotated a few times to yield more information, cf. Fig. 6 b.

Since the problem is ill-posed, we propose to solve the following variational problem to recover $\mathbf{h}$ :

$$
\min _{\mathbf{h} \in \mathbb{R}^{m}} \frac{1}{2}\left\|\left(\mathbf{h} \star \mathbf{y}_{0} \downarrow p\right)-\mathbf{y}_{1}\right\|_{2}^{2}+\frac{\alpha}{2}\|\mathbf{L h}\|_{2}^{2},
$$

where $\alpha \geq 0$ is a regularization parameter and $\mathbf{L}$ is a suitable regularizing operator. In all our experiments we set $\mathbf{L}$ to be the discrete derivative and solve (8) with a linear conjugate gradient descent. The choice of the simplest Tikhonov regularization is to avoid introducing prior information beyond smoothness of the filter. In all our experiments, the parameter $\alpha$ was tuned manually: it is chosen as small as possible while still allowing to avoid the spurious oscillations likely due to noise.

Estimation on simulated data In the synthetic experiment of Fig. 6, we defined $\mathbf{h}$ as an ideal bandlimiting filter, see the blue dashed line in Fig. 6d. We used 64 rotated and projected linear chirps, see Fig. $6 \mathrm{~b}$ and measured the brain phantom from Fig. 4d along them. We also used an alternative spiral [44] based scheme with twice the number of samples of the chirps experiment. We then added $1 \%$ of noise to the measurements and solved the problem (8). The regularization parameter $\alpha$ was tuned manually. The estimation result is shown in Fig. $6 \mathrm{c}$ in the time domain and in Fig. $6 \mathrm{~d}$ in the frequency domain. As can be seen, the chirps based estimation is faithful to the true filter despite some residual noise. The spiral based estimate is far less convincing, though the general shape of the filter in the frequency domain is correct. This shows the importance of choosing a suitable sampling scheme for an accurate estimation of $\mathbf{h}$.

Estimation on experimental data We also evaluated the filter on a 7-Tesla MR scanner (Siemens Healthineers, Erlangen, Germany) with a 1Tx/32Rx head coil (Nova Medical, Wilmington, MA, USA). The maximum gradient amplitude was $80 \mathrm{mT} / \mathrm{m}$ and the maximum slew-rate was $200 \mathrm{~T} / \mathrm{m} / \mathrm{s}$. Here, the minimum dwell-time was $2 \mu \mathrm{s}$. All experiments reported in this paper were conducted using this scanner.

To evaluate the filter, we used 256 chirps on 32 channels. This led to the results depicted in Fig. 7: the filter is rather close to a sinc, with a frequency response that clearly cuts off the high frequencies, and a plateau on the low frequencies. The regularization parameter $\alpha$ was tuned manually to avoid oscillations while still limiting the smoothing due to the quadratic term. Notice that this calibration procedure needs to be performed only once and the obtained filter can be used for every reconstruction trajectory. Hence, we will use the estimated filter in the reconstruction experiments of Section 5.

\section{Handling the filtering effects in reconstruction algorithms}

\subsection{Discretizing the image}

Let $n \in 2 \mathbb{N}$ denote a resolution parameter. In this paper, we assume that the true magnetization $u$ can be written as $u=u_{d} \star \psi$, where

$$
u_{d}=\sum_{-n / 2 \leq i, j<n / 2} \mathbf{u}[i, j] \delta_{i / n, j / n}
$$



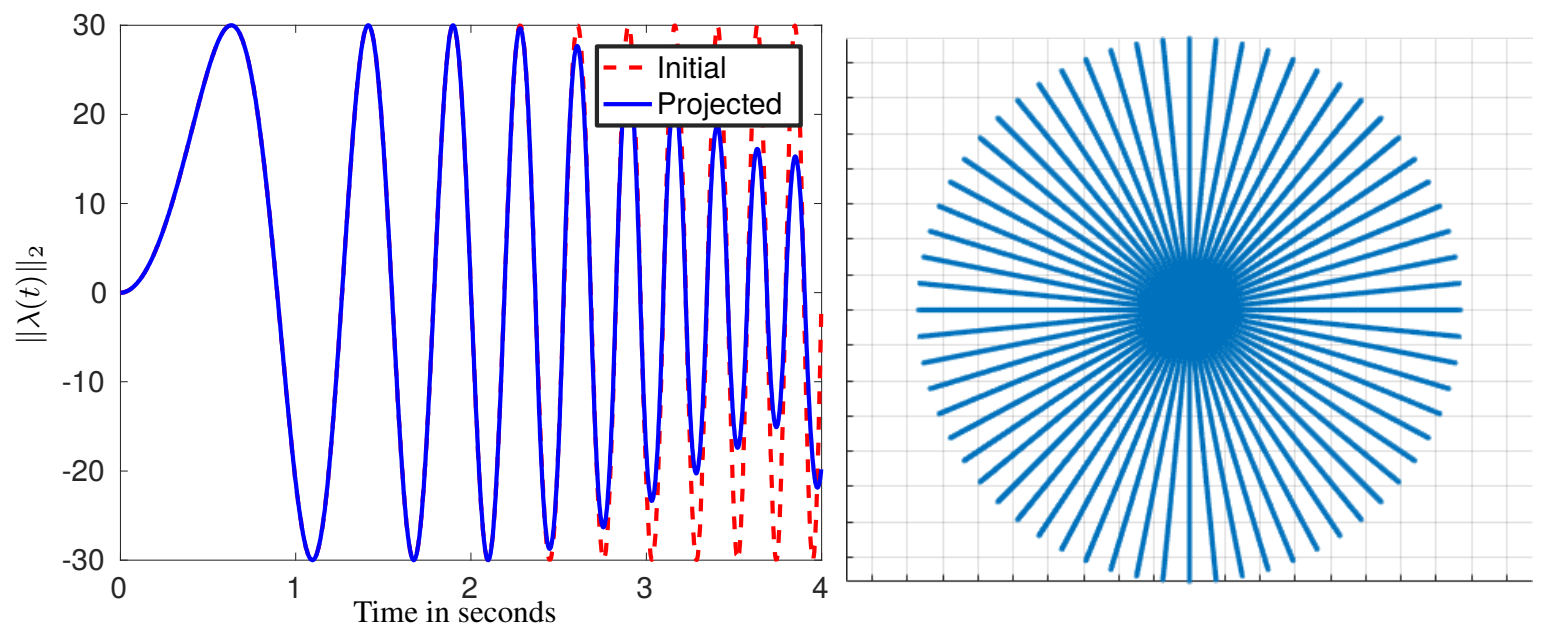

(a) Projected chirp

(b) $k$-space trajectories

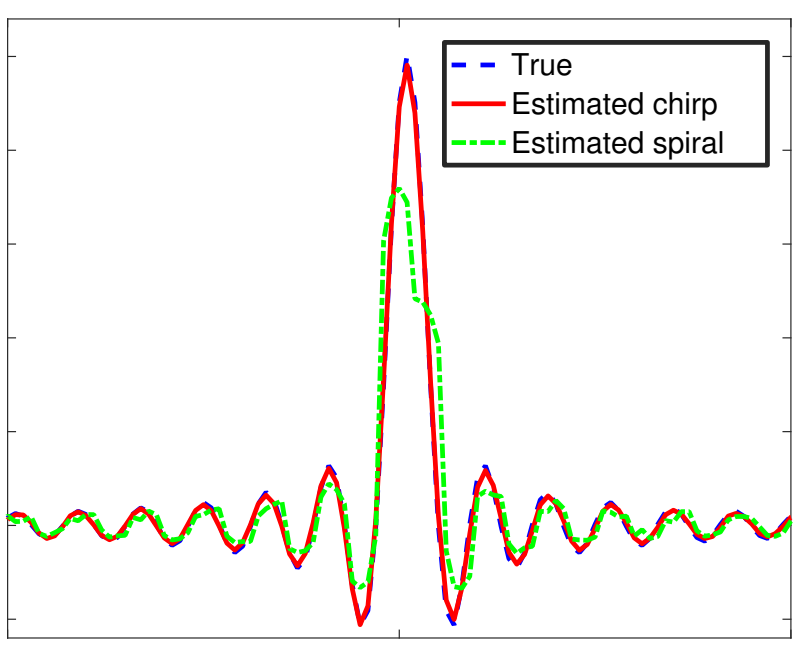

(c) Time response

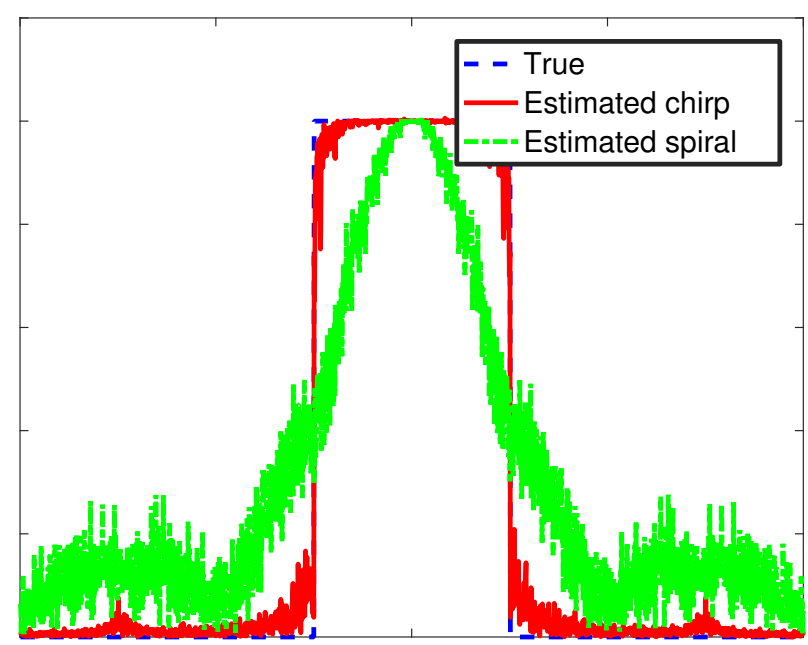

(d) Frequency response

Figure 6: Validation of the estimation procedure. a) the initial chirp and their projection to make the trajectory feasible. b) 64 chirps used to estimate the filter. Each of them crosses the center 20 times. c) The estimation in the time domain. d) The estimation in the frequency domain.

is an atomic discretization of the image with $\mathbf{u} \in \mathbb{C}^{n \times n}$ and $\psi:[-\epsilon, \epsilon]^{2} \rightarrow \mathbb{R}$ is a compactly supported interpolation kernel. The interest of this decomposition lies in the fact that it allows using nonuniform fast Fourier transforms.

The simplest interpolation kernel $\psi$ that will be used in all the experiments of this paper is the spline of order 0 :

$$
\psi(x, y)= \begin{cases}1 & \text { if }-1 /(2 n) \leq x, y<1 /(2 n) \\ 0 & \text { otherwise }\end{cases}
$$

\subsection{Fast implementation of the forward model}

In order to reconstruct the image, almost every reconstruction algorithm requires an implementation of the forward measurement operator and its adjoint. In this section, we therefore propose a numerical algorithm to evaluate integrals 


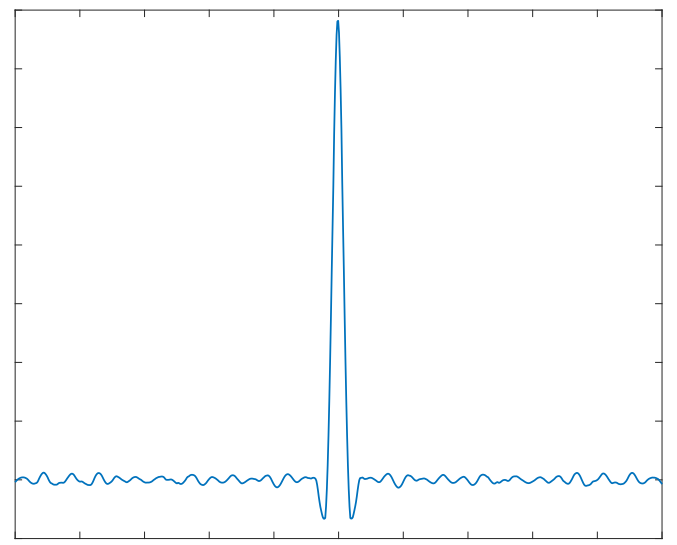

(a) Evaluated filter

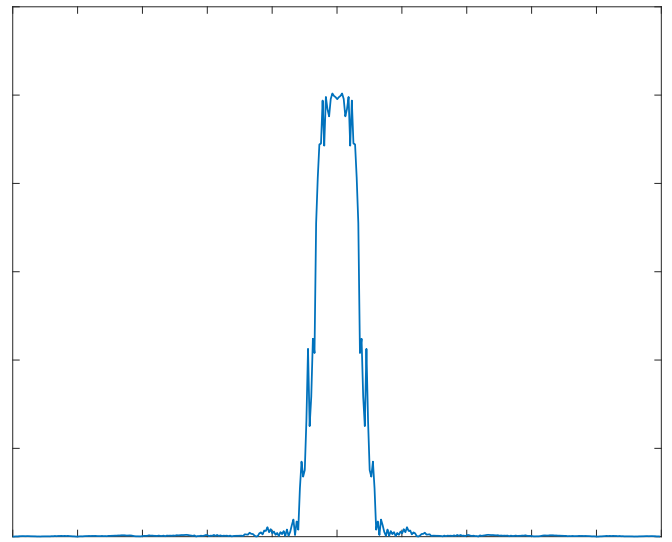

(b) Frequency response

Figure 7: Filter estimated on a real 7T scanner.

of the form:

$$
y_{i}=\int_{\mathbb{R}} h(i \Delta t-t) \hat{u}(\lambda(t)) d t .
$$

It is based on a combination of numerical integration and the nonuniform Fast Fourier transform.

\subsubsection{The NFFT}

The atomic structure in (9) allows using the non uniform Fast Fourier Transform (NFFT or NUFFT) [16, 23] to get a numerical expression of $\hat{u}$ at a set of locations $\mathbf{k}=(\mathbf{k}[0], \ldots, \mathbf{k}[m-1]) \in \mathbb{R}^{m \times 2}$ in the $k$-space. We let $\mathbf{k}_{1}$ and $\mathbf{k}_{2}$ denote the two spatial components of $\mathbf{k}$. The NFFT allows evaluating rapidly with a high precision, all components of $\hat{u}_{d}(\mathbf{k})$, defined by

$$
\hat{u}_{d}(\mathbf{k}[l])=\sum_{-n / 2 \leq i, j<n / 2} \mathbf{u}[i, j] \exp \left(-2 \iota \pi\left(i \mathbf{k}_{1}[l]+j \mathbf{k}_{2}[l]\right)\right) .
$$

While a naive implementation of the sum would require $O\left(m n^{4}\right)$ operations, the NFFT reduces the complexity to $O\left(n^{2} \log (n)+m|\log (\epsilon)|^{2}\right)$, where $\epsilon$ is the desired precision. The constants involved in the $O$ depend on the locations of the sampling points $\mathbf{k}$.

By using a matrix-vector product notation, this can be rewritten as $\hat{u}_{d}(\mathbf{k})=\mathbf{N}_{\mathbf{k}}^{*} \mathbf{u}$, where $\mathbf{N}_{\mathbf{k}}^{*} \in \mathbb{C}^{m \times n^{2}}$ is the NFFT matrix. Then, to get the values of $\hat{u}$, we simply use the formula $\hat{u}=\hat{u}_{d} \odot \hat{\psi}$. Overall, the mapping $(\mathbf{u}, \mathbf{k}) \mapsto \hat{u}(\mathbf{k})=\hat{\mathbf{u}}$ is given by $\hat{\mathbf{u}}=\hat{\psi}(\mathbf{k}) \odot \mathbf{N}_{\mathbf{k}}^{*} \mathbf{u}$.

\subsubsection{Integration along the curve}

The principle In order to compute the integrals (10), we propose to use simple numerical integration procedures. Letting $p \in \mathbb{N}$ denote an upsampling parameter, we shall use the following approximation:

$$
y_{i}=(h \star f)(i \Delta t) \simeq \sum_{j \in \mathbb{Z}} h_{j} \cdot \hat{u}(\lambda(i \Delta t-j \delta t)),
$$

where $\delta t=\Delta t / p$ and where the weights $h_{j}$ can be chosen either by using the estimation procedure proposed in Section 3.1 or taken equal to $h(j \delta t)$ if an analytical version of $h$ is available. More advanced Newton-Cotes formula [38] could also be used. The values $\hat{u}(\lambda(i \Delta t-j \delta t))$ can be evaluated efficiently with the NFFT, and the weights $h_{j}$ only need to be computed once at the start of the algorithm. 
The forward model $\mathbf{A}$ can now be completely described. Letting $\mathbf{k}$ denote the vector of discrete locations with components $\mathbf{k}[i]=\lambda(i \delta t)$ for $0 \leq i \leq m p-1$, it takes the form

$$
\mathbf{A u}=\mathbf{H} \boldsymbol{\Sigma}_{\psi} N_{\mathbf{k}}^{*} \mathbf{u},
$$

where $\mathbf{H}$ is the discrete convolution operator in equation (11) and where $\boldsymbol{\Sigma}_{\psi}: \mathbf{z} \mapsto \hat{\psi}(\mathbf{k}) \odot \mathbf{z}$.

The case of multiple coils In the case of multiple coils, the forward model can be written as

$$
\mathbf{A u}=\left(\mathbf{H} \boldsymbol{\Sigma}_{\psi} \mathbf{N}_{\mathbf{k}}^{*} \mathbf{S}_{1} \mathbf{u}, \ldots, \mathbf{H} \boldsymbol{\Sigma}_{\psi} \mathbf{N}_{\mathbf{k}}^{*} \mathbf{S}_{n_{c}} \mathbf{u}\right)^{T},
$$

where $\mathbf{S}_{k}=\operatorname{diag}\left(\mathbf{s}_{k}\right)$ is the diagonal matrix associated to the $k$-th sensitivity profile and $n_{c}$ is the total number of receiver coils.

How to choose the value of $p$ ? The oversampling factor $p$ should be selected carefully to avoid large computing times while still mitigating the filtering effects. As was explained in Section 2.1, no effect is perceived when the maximal distance between consecutive samples is below $1 / 2$ (half the Nyquist distance on a normalized FOV). Given a trajectory $\lambda$, the maximal distance between two consecutive samples is given by $\Delta_{\max }=\|\dot{\lambda}\|_{\infty} \Delta t$. If $\Delta_{\max }$ is below $1 / 2, p$ can be safely chosen equal to 1 , and the proposed forward model boils down to the standard MRI model. In the other cases, we wish to find the minimum value of $p$ such that $\|\dot{\lambda}\|_{\infty} \delta t \leq 1 / 2$, leading to the choice $p=\left\lceil 2\|\dot{\lambda}\|_{\infty} \Delta t\right\rceil$. In practice, this typically yields values of $p$ in the range $\{1, \ldots, 4\}$.

\subsection{Reconstruction algorithms}

Once the forward operator and its adjoint are properly described, many existing algorithms based on variational principles, such as [37, 31] can be used out of the box. In this work, we make us of simple linear reconstructions based on the linear conjugate gradient method and more advanced nonlinear approaches.

\subsubsection{Estimating the sensitivities}

In all the experiments performed in this paper, we first acquire a reference image with a standard Cartesian trajectory sampled at twice Shannon's rate along the readout direction. This allows to simply estimate the sensitivities by using the sum-of-square approach [33].

\subsubsection{Linear reconstructions}

One of the simplest ways to reconstruct an image is to solve the following Tikhonov-regularized least squares problem:

$$
\min _{\mathbf{u} \in \mathbb{R}^{n}} \frac{1}{2}\|\mathbf{A u}-\mathbf{y}\|_{2}^{2}+\frac{\alpha}{2}\|\mathbf{u}\|_{2}^{2} .
$$

The same formulation is used in the popular reconstruction method SENSE [37]. The optimality conditions for this problem read as $\left(\mathbf{A}^{*} \mathbf{A}+\alpha I\right) \mathbf{u}=\mathbf{A}^{*} \mathbf{y}$, which can be solved with a linear conjugate gradient method.

\subsubsection{Nonlinear reconstructions}

Nonlinear reconstructions are known to yield better results than linear ones, especially in the regime of subsampled data $[6,29,9]$. The whole field of compressed sensing [31, 8], which under certain assumptions guarantees exact reconstructions, is based on $\ell^{1}$-regularized problems. In this paper, our nonlinear reconstructions are based on the resolution of the following formulation:

$$
\min _{\mathbf{u} \in \mathbb{R}^{n}} \frac{1}{2}\|\mathbf{A u}-\mathbf{y}\|_{2}^{2}+\alpha R(\mathbf{u})
$$

where $R: \mathbb{C}^{n^{2}} \rightarrow \mathbb{R} \cup\{+\infty\}$ is a regularization term describing prior information on the image that is sought to be recovered. This type of problem can be solved efficiently by using a Douglas-Rachford algorithm [14]. Letting $f(\mathbf{u})=\frac{1}{2}\|\mathbf{A u}-\mathbf{y}\|_{2}^{2}$, it reads as follows: 


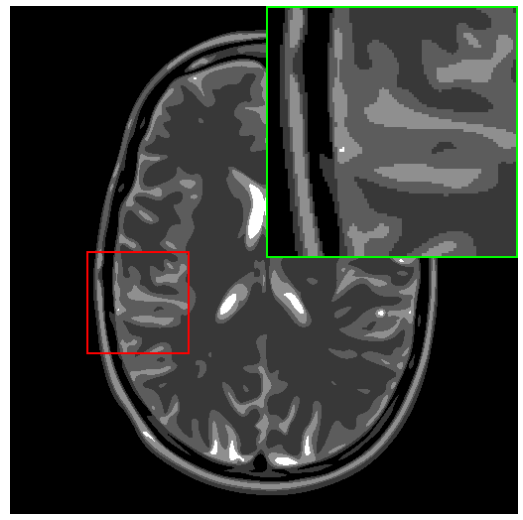

(a) Ground truth

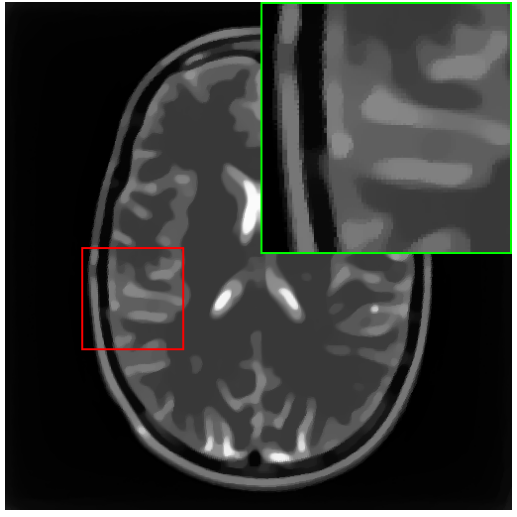

(b) Standard

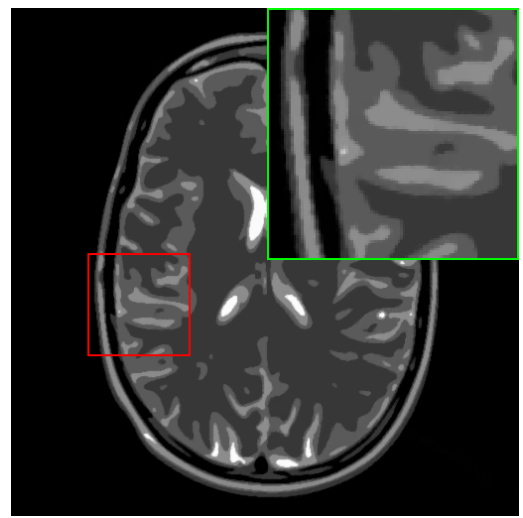

(c) Proposed

Figure 8: Reconstructions of the brain phantom image sampled along spirals without (b) and with (c) accounting for the bandlimiting filtering effects. For each case, we used a total variation based reconstruction with a manually tuned regularization parameter.

1. Input: initial guess $\mathbf{v}_{0} \in \mathbb{C}^{n^{2}}$ and parameter $\gamma>0$.

2. $\mathbf{u}_{k}=\operatorname{Prox}_{\gamma \cdot f}\left(\mathbf{v}_{k}\right)$.

3. $\mathbf{v}_{k+1}=\mathbf{v}_{k}-\mathbf{u}_{k}+\operatorname{Prox}_{\gamma \cdot \alpha \cdot R}\left(2 \mathbf{u}_{k}-\mathbf{v}_{k}\right)$.

The proximal operator $\operatorname{Prox}_{f}$ of a convex function $f$ is thereby defined by

$$
\operatorname{Prox}_{f}(\mathbf{z})=\underset{\mathbf{u} \in \mathbb{C}^{n^{2}}}{\arg \min } f(\mathbf{u})+\frac{1}{2}\|\mathbf{u}-\mathbf{z}\|_{2}^{2} .
$$

Step 2) of the algorithm can be interpreted as the resolution of the inverse problem using Tikhonov regularization. It can be solved using a linear conjugate gradient algorithm. Step 3) can be seen as solving a denoising problem and depending on the choice of the prior $R$, different algorithms can be used. The sequence $\left(\mathbf{u}_{k}\right)_{k \in \mathbb{N}}$ can be shown to converge to a global minimizer of (12) if $R$ is a convex closed function with nonempty interior. In this work, we define $R$ as the total variation of the image [10] and solve the proximal of Step 3) with the method proposed in [43].

\section{Reconstruction results}

In this section, we demonstrate how the proposed reconstruction algorithms perform on simulated and experimental data and compare with results obtained by traditional approaches.

\subsection{Simulated data}

We first consider the case of a sampling pattern based on fast spiral trajectories proposed in $[44,15]$. We designed a sampling pattern made of 128 interleaves. Each interleave lasts $1 \mathrm{~ms}$ and is sampled at the minimum dwell-time of $2 \mu \mathrm{s}$. It is therefore composed of 500 samples. This corresponds to an undersampling factor of 4 . Here, the maximum gradient amplitude was $65 \mathrm{mT} / \mathrm{m}$. During one dwell-time, the traveled k-space distance is therefore $5.53 \mathrm{~m}^{-1}$, which is larger than the Nyquist rate of $4 \mathrm{~m}^{-1}$ (the FOV is $25 \mathrm{~cm}$ ).

We simulated measurements of a brain phantom incorporating the bandlimiting filtering estimated from our $7 \mathrm{~T}$ scanner in Section 3.1. Fig. 8b displays the brain phantom image reconstructed with the nonlinear algorithm of Section 4.3.3 that does not include the filtering in the forward model. Note that this corresponds to a standard MR reconstruction methodology. We reconstructed the same data with the method proposed in Section 4 that accounts for the 
filtering effect. The result is displayed in Fig. 8c. By looking at the magnified regions, we notice that the proposed reconstruction was able to recover the fine structural details present in the ground truth image (Fig. 8a), which had disappeared in the standard reconstruction (Fig. 8b).

In all experiments, the regularization parameter $\alpha$ of equation (12) was manually tuned so as to produce the best possible result from a perceptual point of view. We had to choose it larger for the standard model, since otherwise strong oscillations would have appeared in the reconstructed image, with no significant increase in the level of details.

\subsection{Experimental data}

The proposed acquisition model and reconstruction schemes were tested on experimental data acquired from both spiral [27] and Sparkling [7, 12, 26] sampling patterns. In both cases, the forward operator of Section 4 is based on the filter estimation shown in Fig. 7. Hence, these experiments are based on a real calibration procedure. The regularization parameter $\alpha$ in equation (12) was selected carefully to reduce the effects of the nonlinear regularizer while still removing noise.

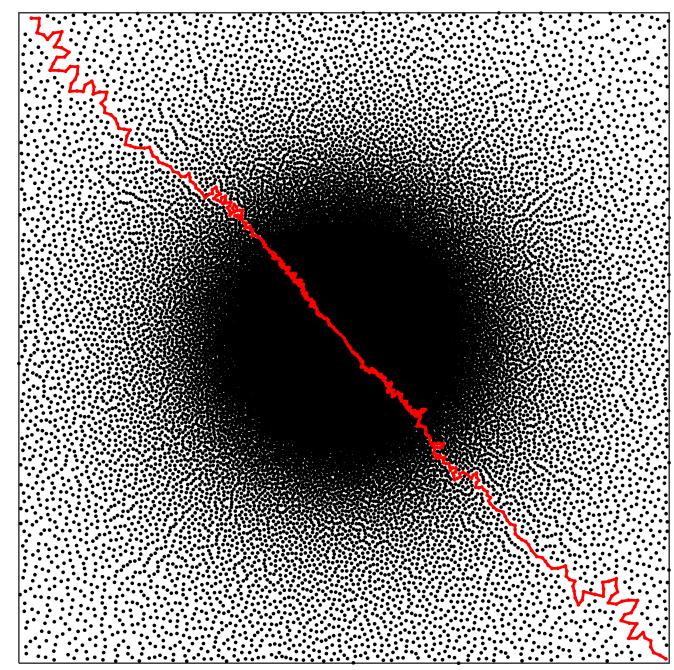

(a) The whole trajectory.

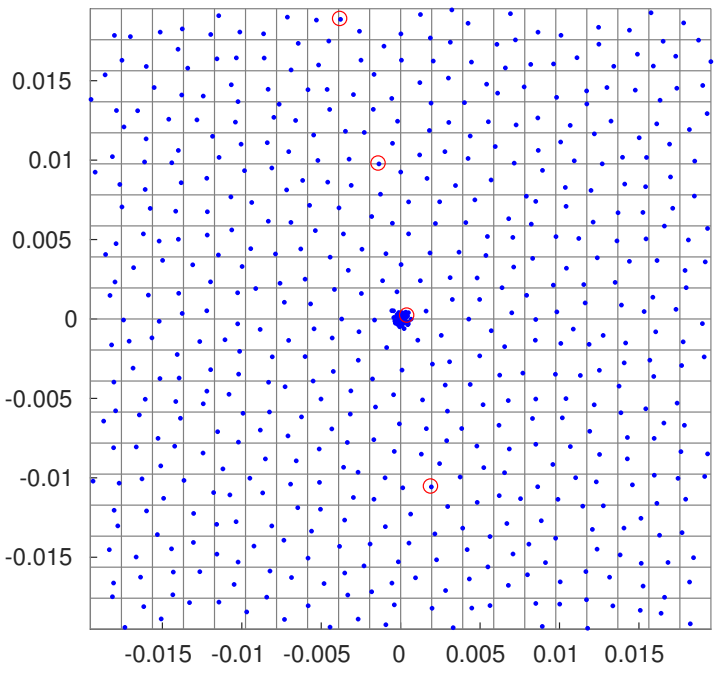

(b) Zoom in the center

Figure 9: Sparkling trajectory and a magnified region of the $k$-space center. Black dots correspond to measured samples and a single shot is depicted in red.

\subsubsection{Spiral sampling}

The spiral trajectory used for the acquisition was the same as in the simulated data of Fig. 8. The reconstruction results are displayed in Fig. 10. In this example, we used a nonlinear reconstruction algorithm. Even though the reconstruction accounting for the filtering effect is not perfect it is clearly superior to a standard reconstruction.

\subsubsection{Sparkling sampling}

The recently introduced Sparkling trajectories are novel non-Cartesian trajectories that produce optimal variabledenisty sampling patterns by taking full advantage of the hardware capacity [7, 12, 26].

The trajectory used in our experiments consisted of 128 shots composed of 512 samples each for a target resolution of $512 \times 512$, corresponding to a subsampling factor of 4 which was chosen based on an empirical study of the maximum acceleration factors in MRI [25]. The proposed sampling scheme allows to keep a high image quality while reducing the scanning time significantly. A typical trajectory is displayed in Fig. 9, where a magnified region of the $k$-space center is displayed in Fig. 9b. As can be seen for the considered trajectory, the distance between consecutive samples is larger than a pixel, be it in the central region or at the $k$-space boundary. 


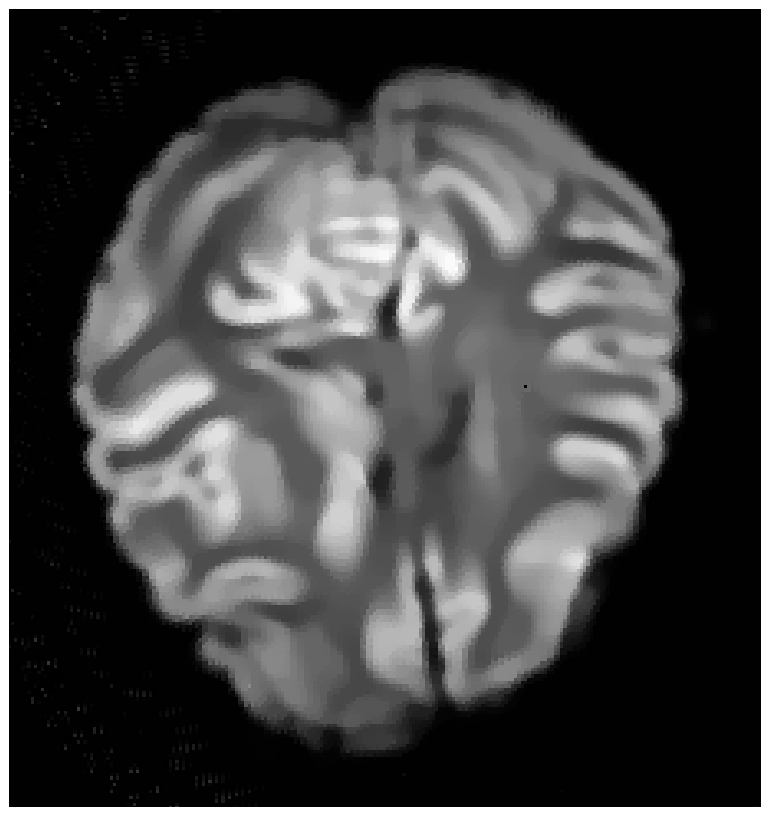

(a) Standard, SNR=19.48dB

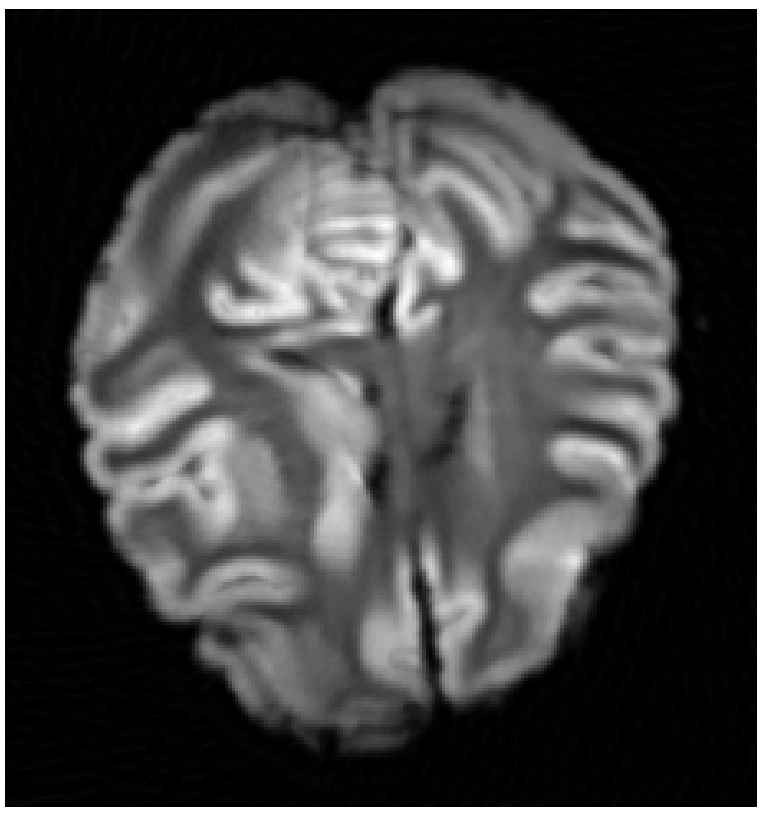

(b) Proposed, SNR=25.62dB

Figure 10: Linear reconstructions of an ex vivo baboon brain acquired with a set of interleaved spiral trajectories, without (a) and with (b) the bandlimiting filtering accounted for. Acquisitions were prospectively performed on a 7 Tesla MR scanner.

An ex vivo human brain was imaged and reconstructed with a total variation based, nonlinear reconstruction algorithm and with the proposed method with $p=4$. The results are displayed in Fig. 11. Once again, the improvement of quality allowed by the proposed approach is striking, especially in the temporal lobes of the brain.

\section{Discussion and Conclusion}

In this work, we illustrated how the anti-aliasing filters implemented in analog-to-digital converters can be detrimental to the reconstruction quality of MRI images. Depending on the speed of the trajectory and on the sampling period, the effect caused by the filters reaches from barely perceptible to drastic and irreversible loss of information. For many trajectories, such effects can be handled quite simply by reducing the dwell-time, however, this might not be a sufficient countermeasure when using fast trajectories which saturate the maximum gradient amplitude. In that case, the proposed numerical algorithms mitigate these effects significantly. The enhanced reconstruction quality comes at the expense of a higher numerical complexity, with computing times typically multiplied by factors ranging from 2 to 4 compared to standard approaches.

The findings of our work might question why such anti-aliasing filters are used in practice. While they make perfect sense when the aim is to reduce the field of view using Cartesian sampling (which is the dominant sampling pattern in commercial systems), their pertinence becomes less obvious with more exotic trajectories and modern nonlinear reconstruction algorithms.

Let us consider their pros and cons. On the positive side, filtering allows to reduce noise and spurious interferences and to increase the signal-to-noise-ratio, which may sometimes be critical when little signal is available. In the case of Cartesian trajectories and bandlimiting filters, it allows to cut-off signal that is beyond the desired field of view. It also allows to reduce the number of measurements and hence leads to faster reconstruction algorithms. In addition, it is physically impossible to measure point-wise values of $f$, and the filtering is an effect that cannot be avoided, up to the temporal resolution of the ADC. On the negative side, applying filters trades temporal resolution for signalto-noise-ratio. It is however well known in the field of inverse problems that it is much harder to gain resolution 


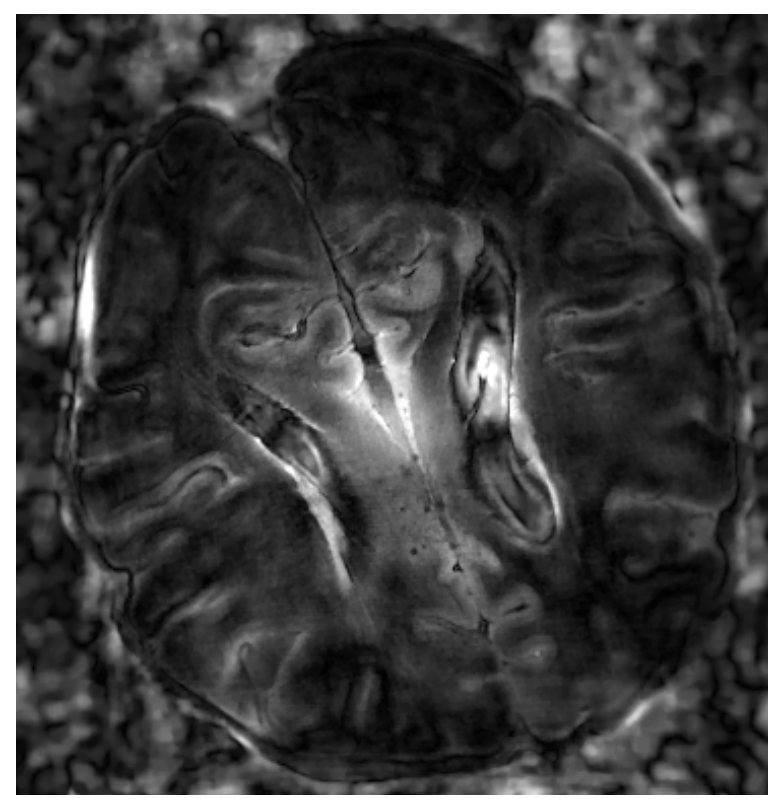

(a) Standard, SNR $=5.45 \mathrm{~dB}$

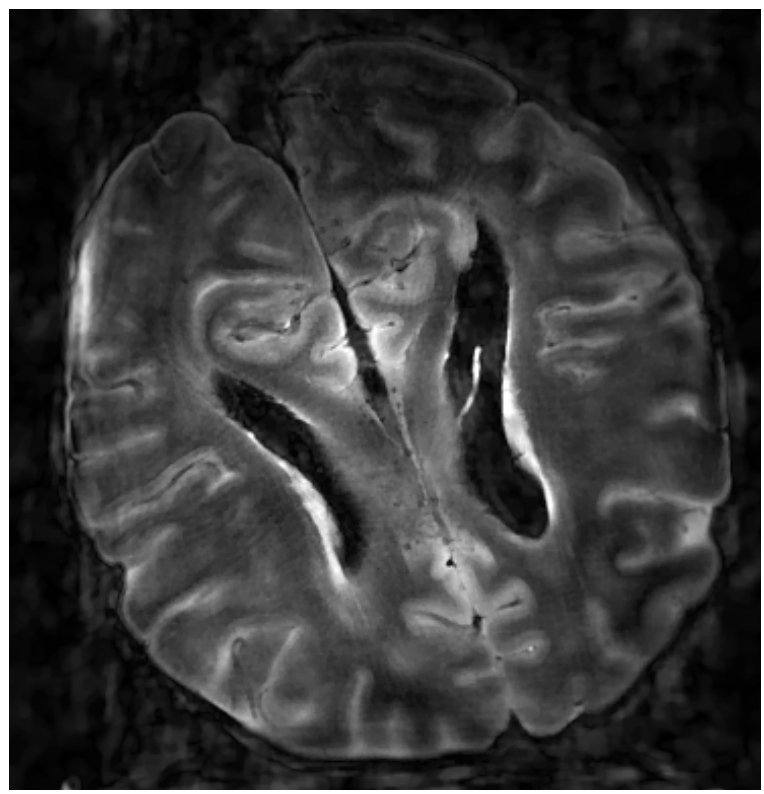

(b) Proposed, SNR=17.38dB

Figure 11: Nonlinear, total variation based reconstructions of ex vivo humain brain with a Sparkling trajectory, without (a) and with (b) the bandlimiting filtering accounted for. Acquisitions were prospectively performed on a 7 Tesla MR scanner.

than signal-to-noise-ratio. State-of-the-art denoisers are suspected to be close to an optimal performance [11], while blurring induces an irreversible loss of information.

Overall, we believe that the increase of computational power using massively parallel architectures, makes the arguments supporting the reduction of data partly irrelevant. A striking side-result of this work is that the older technology of integrating ADC is somewhat preferable to more complex bandlimiting ADCs, since the integrating filters do not cause an irreversible loss of information when using the proposed algorithms (compare Fig. 1 and Fig. 2). In addition, we speculate that it may be beneficial to use time-varying sampling periods. In the center of the $k$-space, a lot of signal is usually available, allowing to use very short sampling periods. On the contrary, as the sampling trajectory gets more distant to the center, the sampling period could be increased in order to retrieve information above the quantization level despite the signal decay.

The key factor to know whether a trajectory is acceptable or not for standard reconstruction methods is the maximal distance between consecutive samples. Nearly no effect should be observed below half a pixel, slight effects will be observed between half a pixel and 1.5 pixels, and significant problems should appear beyond. The recent advances in sampling theory advocate the use of more complex trajectories with high speeds $[42,32,2,26]$ that may cross the critical regime of sampling distances. In particular, Weiger et al. use a gradient amplitude of $200 \mathrm{mT} / \mathrm{m}$ [42], which would necessitate a sampling bandwidth of $B W=2 \gamma \cdot \operatorname{diam}(\Omega) \cdot G_{\max }=4.25 \mathrm{MHz}$ for a standard field-of-view of $25 \mathrm{~cm}$. This is far above the maximum sampling rate offered by the current MR vendors, which do not go beyond $1 \mathrm{MHz}$ to the best of our knowledge. Those arguments make us believe that the proposed analysis and algorithmic framework should be taken under consideration in the development of sequences and MR scanners.

On the side of mathematics, we believe that this paper offers new challenges in sampling theory. The experiments in this paper show that the sampling model (6) is realistic and accurate. Surprisingly though, we are not aware of a theory allowing to explain why and how this model leads to stable reconstructions.

Acknowledgements C. Lazarus and P. Weiss wish to acknowledge P. Ciuciu and A. Vignaud for helping them to identify the filtering effect thanks to their strong engagement in the acquisition of Sparkling sampling patterns. M. März and P. Weiss wish to warmly thank G. Kutyniok for her support and enabling them to meet on a regular basis. 
The authors wish to thank David Brünner for sending them some references regarding the receive chain in MRI. We would like to express our gratitude to the donors involved in the body donation program of the Association des dons $d u$ corps $d u$ Centre Ouest, Tours, who made it possible to use an ex vivo human brain as phantom for our experiments, by generously donating their bodies for science. We also would like to acknowledge C. Destrieux and I. Zemmoura for the extraction and fixation of this anatomical piece. We also wish to thank M. Bottlaender for providing the baboon phantom. C. Lazarus wishes to thank the CEA Irtelis international PhD program for its financial support. She also acknowledges the France Life Imaging project Multi-CS-MRI, which supported her traveling expenses to ITAV. M. März is supported by the DFG Priority Program DFG-SPP 1798. P. Weiss thanks the ANR JCJC OMS for partial funding.

\section{References}

[1] N. O. Addy, H. H. Wu, and D. G. Nishimura. Simple method for MR gradient system characterization and k-space trajectory estimation. Magnetic Resonance in Medicine, 68(1):120-129, 2012.

[2] C. B. Ahn, J. H. Kim, and Z. H. Cho. High-Speed Spiral-Scan Echo Planar NMR Imaging-I. IEEE Transactions on Medical Imaging, 5(1):2-7, 1986.

[3] Akram Aldroubi. Non-uniform weighted average sampling and reconstruction in shift-invariant and wavelet spaces. Applied and Computational Harmonic Analysis, 13(2):151-161, 2002.

[4] R. Ansorge and M. J. Graves. The Physics and Mathematics of MRI. Morgan \& Claypool Publishers, 2016.

[5] R. Behin, J. Bishop, and R. M. Henkelman. Dynamic range requirements for MRI. Concepts in Magnetic Resonance Part B: Magnetic Resonance Engineering: An Educational Journal, 26(1):28-35, 2005.

[6] K. T. Block, M. Uecker, and J. Frahm. Undersampled radial MRI with multiple coils. Iterative image reconstruction using a total variation constraint. Magnetic Resonance in Medicine, 57(6):1086-1098, 2007.

[7] C. Boyer, N. Chauffert, P. Ciuciu, J. Kahn, and P. Weiss. On the Generation of Sampling Schemes for Magnetic Resonance Imaging. SIAM Journal on Imaging Sciences, 9(4):2039-2072, 2016.

[8] E. J. Candès, J. Romberg, and T. Tao. Robust uncertainty principles: exact signal reconstruction from highly incomplete frequency information. IEEE Transactions on information theory, 52(2):489-509, 2006.

[9] L. Chaâri, J.-C. Pesquet, A. Benazza-Benyahia, and P. Ciuciu. A wavelet-based regularized reconstruction algorithm for SENSE parallel MRI with applications to neuroimaging. Medical image analysis, 15(2):185-201, 2011.

[10] A. Chambolle, V. Caselles, D. Cremers, M. Novaga, and T. Pock. An introduction to total variation for image analysis. Theoretical Foundations and Numerical Methods for Sparse Recovery, 9(263-340):227, 2010.

[11] P. Chatterjee and P. Milanfar. Is Denoising Dead? IEEE Transactions on Image Processing, 19(4):895-911, 2010.

[12] N. Chauffert, P. Ciuciu, J. Kahn, and P. Weiss. A Projection Method on Measures Sets. Constructive Approximation, 45(1):83-111, 2017.

[13] N. Chauffert, P. Weiss, J. Kahn, and P. Ciuciu. A projection algorithm for gradient waveforms design in Magnetic Resonance Imaging. IEEE transactions on medical imaging, 35(9):2026-2039, 2016.

[14] P. L. Combettes and J.-C. Pesquet. Proximal Splitting Methods in Signal Processing. In Fixed-Point Algorithms for Inverse Problems in Science and Engineering, pages 185-212. Springer, 2011.

[15] B. Delattre, R. M. Heidemann, L. A. Crowe, J.-P. Vallée, and J.-N. Hyacinthe. Spiral demystified. Magnetic Resonance Imaging, 28(6):862-881, 2010. 
[16] A. Dutt and V. Rokhlin. Fast Fourier Transforms for Nonequispaced Data. SIAM Journal on Scientific computing, 14(6):1368-1393, 1993.

[17] J. A. Fessler. Model-Based Image Reconstruction for MRI. IEEE Signal Processing Magazine, 27(4):81-89, 2010.

[18] J. Graessner. Bandwidth in MRI. Magnetom Flash, 2:3-8, 2013.

[19] M. Guerquin-Kern, L. Lejeune, K. P. Pruessmann, and M. Unser. Realistic analytical phantoms for parallel magnetic resonance imaging. IEEE Transactions on Medical Imaging, 31(3):626-636, 2012.

[20] D. I. Hoult. The nmr receiver: A description and analysis of design. Progress in Nuclear Magnetic Resonance Spectroscopy, 12(1):41-77, 1978.

[21] D. I. Hoult. Receiver design for MR. eMagRes, pages 1-21, 2007.

[22] M. Jouda, O. G. Gruschke, and J. G. Korvink. A new fully integrated multichannel receiver design for magnetic resonance imaging. Concepts in Magnetic Resonance Part B: Magnetic Resonance Engineering, 46(3):134-145, 2016.

[23] J. Keiner, S. Kunis, and D. Potts. Using NFFT 3-A Software Library for Various Nonequispaced Fast Fourier Transforms. ACM Transactions on Mathematical Software (TOMS), 36(4):19, 2009.

[24] P. C. Lauterbur. Image Formation by Induced Local Interactions: Examples Employing Nuclear Magnetic Resonance. Nature Publishing Group, 1973.

[25] C. Lazarus, P. Weiss, A. Vignaud, and P. Ciuciu. An empirical study of the maximum degree of undersampling in compressed sensing for T2*-weighted MRI. Magnetic Resonance Imaging, 53:112-122, 2018.

[26] Carole Lazarus, Pierre Weiss, Nicolas Chauffert, Franck Mauconduit, Loubna El Gueddari, Christophe Destrieux, Ilyess Zemmoura, Alexandre Vignaud, and Philippe Ciuciu. Sparkling: variable-density k-space filling curves for accelerated t $2 *$-weighted mri. Magnetic resonance in medicine, 81(6):3643-3661, 2019.

[27] J. H. Lee, B. A. Hargreaves, B. S. Hu, and D. G. Nishimura. Fast 3D imaging using variable-density spiral trajectories with applications to limb perfusion. Magnetic Resonance in Medicine, 50(6):1276-1285, 2003.

[28] Jin Hyung Lee, Greig C Scott, John M Pauly, and Dwight G Nishimura. Broadband multicoil imaging using multiple demodulation hardware: A feasibility study. Magnetic Resonance in Medicine: An Official Journal of the International Society for Magnetic Resonance in Medicine, 54(3):669-676, 2005.

[29] D. Liang, B. Liu, J. Wang, and L. Ying. Accelerating sense using compressed sensing. Magnetic Resonance in Medicine, 62(6):1574-1584, 2009.

[30] RB Lufkin, E Pusey, DD Stark, R Brown, B Leikind, and WN Hanafee. Boundary artifact due to truncation errors in mr imaging. American Journal of Roentgenology, 147(6):1283-1287, 1986.

[31] M. Lustig, D. Donoho, and J. M. Pauly. Sparse MRI: The application of compressed sensing for rapid MR imaging. Magnetic Resonance in Medicine, 58(6):1182-1195, 2007.

[32] P. Mansfield. Multi-planar image formation using NMR spin echoes. Journal of Physics C: Solid State Physics, 10(3), 1977.

[33] C. A. McKenzie, E. N. Yeh, M. A. Ohliger, M. D. Price, and D. K. Sodickson. Self-calibrating parallel imaging with automatic coil sensitivity extraction. Magnetic Resonance in Medicine, 47(3):529-538, 2002.

[34] ER McVeigh, RM Henkelman, and MJ Bronskill. Noise and filtration in magnetic resonance imaging. Medical physics, 12(5):586-591, 1985. 
[35] C. H. Meyer, B. S. Hu, D. G. Nishimura, and A. Macovski. Fast spiral coronary artery imaging. Magnetic Resonance in Medicine, 28(2):202-213, 1992.

[36] Mila Nikolova. Analysis of the recovery of edges in images and signals by minimizing nonconvex regularized least-squares. Multiscale Modeling \& Simulation, 4(3):960-991, 2005.

[37] K. P. Pruessmann, M. Weiger, M. B. Scheidegger, and P. Boesiger. Sense: sensitivity encoding for fast mri. Magnetic Resonance in Medicine, 42(5):952-962, 1999.

[38] A. Ralston and P. Rabinowitz. A First Course in Numerical Analysis. Courier Corporation, 2001.

[39] C. E. Shannon. A Mathematical Theory of Communication. The Bell System Technical Journal, 27(3):379-423, 1948.

[40] W. Tang, W. Wang, W. Liu, Y. Ma, X. Tang, L. Xiao, and J.-H. Gao. A home-built digital optical MRI console using high-speed serial links. Magnetic Resonance in Medicine, 74(2):578-588, 2015.

[41] A. Tarantola. Inverse Problem Theory and Methods for Model Parameter Estimation, volume 89. SIAM, 2005.

[42] M. Weiger, J. Overweg, M. B. Rösler, R. Froidevaux, F. Hennel, B. J. Wilm, A. Penn, U. Sturzenegger, W. Schuth, M. Mathlener, et al. A high-performance gradient insert for rapid and short-T2 imaging at full duty cycle. Magnetic Resonance in Medicine, 2017.

[43] P. Weiss, L. Blanc-Féraud, and G. Aubert. Efficient Schemes for Total Variation Minimization Under Constraints in Image Processing. SIAM Journal on Scientific Computing, 31(3):2047-2080, 2009.

[44] T Zhao, Y Qian, YK Hue, T Ibrahim, and F Boada. An improved analytical solution for variable density spiral design. In Proceedings 16th Scientific Meeting, International Society for Magnetic Resonance in Medicine, Toronto, page 1342, 2008. 